\title{
Correlates of Depression among US Military Members Infected with the Human Immunodeficiency Virus
}

Robert J. Carpenter ${ }^{1 *}$, Mark S. Riddle ${ }^{2}$, Dennis A. White ${ }^{3}$ and Anuradha Ganesan ${ }^{4}$

${ }^{1}$ Infectious Diseases Staff Physician, Division of Infectious Diseases, Naval Medical Center San Diego, 34800 Bob Wilson Drive, San Diego, CA, 92134, USA

${ }^{2}$ Investigator, Enteric Diseases Department, Naval Medical Research Center, 503 Robert Grant Ave, Silver Spring, MD, 20910, USA

${ }^{3}$ Staff Psychiatrist, 7th Marine Regiment, United States Marine Corps, USA

${ }^{4}$ Infectious Diseases Staff Physician, Department of Infectious Diseases, Walter Reed National Military Medical Center; Infectious Diseases Clinical Research Program, Uniformed Services University of the Health Sciences, 8901 Rockville Pike, Bethesda, MD, 20889, USA

\section{Abstract}

Objective(s): To define the incidence of mental health disorders (MHD) and risk factors associated with incident MHD in an HIV clinic setting with unrestricted access to care and limited confounding due to concomitant injection drug use (IDU).

\section{Design: Retrospective cohort study.}

Methods: Eligible subjects were HIV positive adults followed at a US military treatment facility during the calendar year 2005. Incidence rates are calculated per 100 person years (py). Cox proportional hazards regression were utilized to identify risk factors associated with MHD. All persons not experiencing MHD were followed until study completion in January 2008.

Results: 494 subjects ( $51 \%$ Black, 93\% male, median CD4 count 458) were followed for 5200 py. 160 subjects developed MHD for an overall incidence of 3.1/100 py of follow up. The incidence of depression and anxiety were $2.4 / 100$ py and $0.4 / 100$ py, respectively. Factors associated with a diagnosis of incident MHD include receipt of a protease inhibitor (PI) based regimen (HR 2.0), non-Black ethnicity, and HIV diagnosis in the post HAART era; male gender was protective.

Conclusion: In a cohort with limited confounding, incident MHD was common. Unlike prior studies, nonnucleoside reverse transcriptase inhibitor (NNRTI) use and MHD association was not observed; instead PI use was associated with MHD, perhaps due to PI enhanced mitochondrial toxicity, signaling need for further research. Given the significant burden of MHD observed in this cohort, mental health screening should be considered in the management of HIV infection.

Keywords: HIV; Mental health; Antiretroviral agents; Depression; Anxiety; Military

\section{Introduction}

Potent Antiretroviral Therapy (ART) has transformed HIV infection into a manageable chronic disease. However, ART use is not without potential adverse effects and has been associated with a variety of dysmetabolic syndromes, including hyperlipidemia, hyperglycemia, hepatitis, and coronary artery disease [1]. There is also conflicting evidence that ART may exacerbate existing mental health disorders (MHD) [2-5].

Rates of MHD among HIV infected persons have been found to be highly variable, ranging from $9.4 \%$ to $37 \%$ and are two to three times higher than the rates observed in comparable HIV negative populations [6-10]. Incidence estimates may in fact be underestimates due to ascertainment bias as Asch et al. report that $45 \%$ of HIV infected persons with major depression are undiagnosed [11]. In general, the presence of concomitant MHD has been associated with increased morbidity and mortality [12-14]. Investigators have studied the effects of ART on depressive symptoms, however, the results have been heterogeneous and these studies have tended to include a patient population who were also injection drugs users (IDU), an independent risk factor for MHD and a potentially significant confounder $[15,16]$.

The US military follows a cohort of HIV infected Department of Defense beneficiaries. Due to mandatory drug screening policies, IDU in this cohort is negligible [17]. Additionally, members of this cohort enjoy equal and open access to medical care and medications without copayments; therefore, access to care confounders are likely eliminated in the study of this cohort. To our knowledge, no study has evaluated the incidence of MHD in such a unique cohort and we were specifically interested in examining whether members of this cohort who are treated with ART suffer with MHD at a higher rate than those who are not treated with ART.

\section{Materials and Methods}

\section{Patient population}

HIV infected persons in the US Navy are evaluated semi-annually at one of three military treatment facilities, namely Walter Reed National Military Medical Center (WRNMMC), Bethesda, MD, Naval Medical Center San Diego, CA, or Naval Medical Center Portsmouth, VA. All US Navy beneficiaries enjoy equal and open access to medication and

*Corresponding author: Robert J. Carpenter, Infectious Diseases Staff Physician, Division of Infectious Diseases, Naval Medical Center San Diego, 34800 Bob Wilson Drive, San Diego, CA, 92134, USA, Tel: 619-532-7475; Fax: 619-532-7478; E-mail: rjc311@gmail.com

Received March 31, 2012; Accepted April 17, 2012; Published April 21, 2012

Citation: Carpenter RJ, Riddle MS, White DA, Ganesan A (2012) Correlates of Depression among US Military Members Infected with the Human Immunodeficiency Virus. J AIDS Clinic Res 3:149. doi:10.4172/2155-6113.1000149

Copyright: @ 2012 Carpenter RJ, et al. This is an open-access article distributed under the terms of the Creative Commons Attribution License, which permits unrestricted use, distribution, and reproduction in any medium, provided the original author and source are credited. 
care. A retrospective review of medical records of HIV infected persons followed at WRNMMC during the calendar year 2005 was performed. Subjects were eligible for study if they were HIV infected for two or more years and older than 18 years of age. In order to minimize selection bias, medical records of all patients who reported for HIV clinical or research visits over a 12-month period, between January and December 2005, were reviewed.

\section{Data collected}

After appropriate training, two physicians reviewed outpatient paper and electronic medical records using a predefined data collection sheet. Demographic data collected included: age at HIV diagnosis, gender, race, and military rank. HIV specific data collected: date of positive HIV antibody test and all antiretroviral prescription history including specific drugs and start/stop dates of each. HIV diagnosis was divided into pre HAART (prior to 1996), early post HAART (1996-2000), and late post HAART (after 2000) era. MHD data collected: MHD and date of diagnosis. MHD was defined as any axis I diagnosis as delineated by DSM-IV. Diagnosis made by any physician and recorded in the medical record was accepted; however, DMS-IV criteria were not requisites in recording a MHD. Antidepressant/anxiolytic prescriptions or referrals for mental health evaluation were not classified as an MHD if a corresponding MHD diagnosis was not recorded in the medical record.

\section{Outcomes}

The primary objective of the study was to describe the incidence of MHD among non-IDU, HIV infected persons on and off of ART. Secondary objectives were to examine whether or not HIV infected persons treated with ART suffer with MHD at a higher rate than those who are not treated with ART and whether any particular antiretroviral regimen was associated with MHD.

\section{Statistics}

Descriptive statistics were used to describe the population under study. Proportions were compared using chi-square tests or Fisher's exact tests as appropriate. Incidence rates were calculated per 100 person years of follow up (py), with exact Poisson 95\% confidence intervals (CI). Follow up time was calculated from the time of HIV diagnosis to the time of censoring, defined as the time of an incident MHD or at last follow up prior to January $1^{\text {st }} 2008$. Cox proportional hazards methods were used to model associations between factors and incident MHD. Covariates evaluated included age at diagnosis, gender race/ethnicity, military rank, CD4 count at censor, viral load at censor, ARV use at censor, and period of HIV diagnosis (on or before 1996, between 1996-2000, after 2000).

A multivariable model was developed to evaluate the association between the number of MHD events and important covariates using a backward elimination approach. Variables with a univariate $\mathrm{p}<$ 0.20 were initially added to the model and removed iteratively until all variables were significant at a two-sided alpha $=0.15$. Significance for the multivariate models was defined at alpha $<0.05$. All $\mathrm{p}$ values are 2-sided. Hazard ratios (HRs) and rates are presented with $95 \%$ confidence intervals (95\% CIs). All analyses were conducted using Stata software, version 11 (Statacorp, College Station, TX).

\section{Results}

Four-hundred ninety-four (83\%) of 593 screened subjects met inclusion criteria and were followed for 5200 person years. Excluded subjects included 86 who had missing records, 3 were $<18$ years of age, 6 were HIV-infected for $<2$ years, and 4 had a preexisting axis I diagnosis.
Subjects were predominantly enlisted (86\%) male and were ethnically diverse (Black 51\%, Caucasian 40\%). At censor, the median CD4 count was 458 cells/ $\mu \mathrm{L}$ and viral load was 3954 copies/mL (Table 1).

One-hundred sixty subjects developed an axis I diagnosis with incidences: pre HAART 2.42/100 py, early post HAART 4.16/100 py, and late post HAART 7.16/100 py (3.08/100 py overall). Depression was the most common diagnosis, observed in 123 (25\%) subjects; incidences for depression: pre HAART 1.94/100 py, early post HAART 3.39/100 py, and late post HAART 4.77/100 py. Twenty (4\%) received a diagnosis of anxiety disorder; incidences for anxiety: pre HAART $0.29 / 100$ py, early post HAART $0.17 / 100$ py, and late post HAART $1.39 / 100$ py (Table 2).

Of the 160 subjects with an axis I diagnosis, 64 (40\%) subjects were receiving ART and of the 334 patients without an axis I diagnosis, 81 (22\%) were receiving ART.

In a multivariate analysis, factors associated with depression included Caucasian race (HR 1.7, 95\% CI 1.2-2.6), enlisted rank (HR 2.5, 95\% CI 1.1-5.7), and diagnosis of HIV in the post HAART era (early HR 2.2, 95\% CI 1.3-3.8; late HR 3.9, 95\% CI 2.2-7.0); male gender was protective (HR $0.28,95 \%$ CI $0.1-0.7$ ), ART use was associated with an increased MHD risk (axis I HR 1.9, 95\% CI 1.3-2.7; and depression HR 2.4, 95\% CI 1.6-3.4); whereas a diagnosis of anxiety was not associated with ART use (Table 2).

With respect to ART, protease inhibitor (PI) therapy (HR 2.4, 95\% CI 1.5-4.0) increased depression risk more than non-nucleoside reverse transcriptase inhibitor (NNRTI) therapy (HR 1.6, NS). Factors associated with anxiety were Caucasian and Hispanic/Latino race (HR 3.2, 95\% CI 1.1-9.4; HR 5.6, 95\% CI 1.1-29.4, respectively) and HIV diagnosis during late post HAART era (HR 8.7, 95\% CI 1.8-42.0). Gender, military rank, and ART were not associated with anxiety (Table 2).

\begin{tabular}{|l|l|}
\hline HIV variables & \\
\hline Median CD4 count at censor & 458 \\
\hline Median HIV VL at censor & 3954 \\
\hline Antiretroviral status & $\mathbf{n}(\%)$ \\
\hline Receiving ART at censor & $145(29)$ \\
\hline Not receiving ART at censor & $349(71)$ \\
\hline HIV diagnosis period & \\
\hline Prior to 1996 & $262(53)$ \\
\hline 1996-2000 & $105(21)$ \\
\hline After 2000 & $127(26)$ \\
\hline Demographic variables & \\
\hline Gender & \\
\hline Male & $460(93)$ \\
\hline Female & $34(7)$ \\
\hline Military Rank & \\
\hline Enlisted & $423(86)$ \\
\hline Officer & $46(9)$ \\
\hline Civilian & $25(5)$ \\
\hline Race & \\
\hline Black & $253(51)$ \\
\hline Asian & $10(2)$ \\
\hline Caucasian & $196(40)$ \\
\hline Hispanic/Latino & $30(6)$ \\
\hline Other & $5(1)$ \\
\hline VL plasma viral & \\
\hline
\end{tabular}

$\mathrm{VL}=$ plasma viral load

ART = antiretroviral therapy

Table 1: HIV-infected cohort characteristics, 2005 - Bethesda, Maryland, N=494. 


\begin{tabular}{|c|c|c|c|c|c|c|c|c|c|}
\hline & \multicolumn{3}{|c|}{$\begin{array}{c}\text { Axis I, } \mathrm{n}=160(32 \%) \text { Incidence (cases } / 100 \\
\text { py) }\end{array}$} & \multicolumn{3}{|c|}{$\begin{array}{c}\text { Depression, } n=123(25 \%) \text { Incidence } \\
\text { (cases/100 py) }\end{array}$} & \multicolumn{3}{|c|}{$\begin{array}{c}\text { Anxiety, } n=20(4 \%) \text { Incidence (cases } / 100 \\
\text { py) }\end{array}$} \\
\hline pre HAART $(n=290)$ & \multicolumn{3}{|c|}{2.42} & \multicolumn{3}{|c|}{1.94} & \multicolumn{3}{|c|}{0.29} \\
\hline early post HAART $(n=77)$ & \multicolumn{3}{|c|}{4.16} & \multicolumn{3}{|c|}{3.39} & \multicolumn{3}{|c|}{0.17} \\
\hline \multirow{2}{*}{ late post HAART $(n=127)$} & \multicolumn{3}{|c|}{7.16} & \multicolumn{3}{|c|}{4.77} & \multicolumn{3}{|c|}{1.39} \\
\hline & HR & $95 \% \mathrm{Cl}$ & p & HR & $95 \% \mathrm{Cl}$ & $\mathbf{p}$ & HR & $95 \% \mathrm{Cl}$ & $\mathbf{p}$ \\
\hline \multicolumn{10}{|l|}{ Gender } \\
\hline Male & 0.36 & $(0.16-0.84)$ & 0.018 & 0.28 & $(0.11-0.71)$ & 0.007 & 0.19 & $(0.02-1.66)$ & 0.135 \\
\hline Female & 1 & & & 1 & & & 1 & & \\
\hline \multicolumn{10}{|l|}{ Military Rank } \\
\hline Civilian & 0.99 & $(0.29-3.32)$ & 0.986 & 1.01 & $(0.26-3.94)$ & 0.992 & 0.73 & $(0.04-12.82)$ & 0.828 \\
\hline Officer & 1 & & & 1 & & & 1 & & \\
\hline Enlisted & 1.85 & $(0.93-3.69)$ & 0.082 & 2.47 & $(1.07-5.71)$ & 0.034 & 0.89 & $(0.24-3.27)$ & 0.857 \\
\hline \multicolumn{10}{|l|}{ Race } \\
\hline Black & 1 & & & 1 & & & 1 & & \\
\hline Asian & 1.13 & $(0.27-4.81)$ & 0.867 & 1.54 & $(0.36-6.60)$ & 0.564 & - & & \\
\hline Caucasian & 1.65 & $(1.15-2.37)$ & 0.006 & 1.74 & $(1.18-2.55)$ & 0.005 & 3.20 & $(1.09-9.37)$ & 0.034 \\
\hline Hispanic/Latino & 2.02 & $(1.05-3.89)$ & 0.035 & 1.90 & $(0.92-3.90)$ & 0.082 & 5.56 & $(1.05-29.39)$ & 0.043 \\
\hline \multicolumn{10}{|c|}{ Antiretroviral status at censor } \\
\hline None & 1 & & & 1 & & & 1 & & \\
\hline Yes & 1.89 & $(1.34-2.68)$ & $<0.001$ & 2.35 & $(1.62-3.41)$ & $<0.001$ & 1.63 & $(0.59-4.51)$ & 0.346 \\
\hline NNRTI backbone & 1.31 & $(0.78-2.22)$ & 0.311 & 1.63 & $(0.94-2.82)$ & 0.083 & 0.38 & $(0.05-3.04)$ & 0.360 \\
\hline PI backbone & 1.98 & $(1.24-3.14)$ & 0.004 & 2.44 & $(1.47-4.03)$ & 0.001 & 2.41 & $(0.61-9.54)$ & 0.209 \\
\hline \multicolumn{10}{|l|}{ HIV diagnosis period } \\
\hline Prior to 1996 & 1 & & & 1 & & & 1 & & \\
\hline $1996-2000$ & 2.18 & $(1.28-3.70)$ & 0.004 & 2.22 & $(1.28-3.84)$ & 0.004 & 0.98 & $(0.11-8.55)$ & 0.989 \\
\hline After 2000 & 5.02 & $(2.95-8.55)$ & $<0.001$ & 3.91 & $(2.18-7.00)$ & $<0.001$ & 8.73 & $(1.81-42.01)$ & 0.007 \\
\hline
\end{tabular}

HAART = highly active antiretroviral therapy; NNRTI = non-nucleoside reverse transcriptase inhibitor; $\mathrm{PI}=$ protease inhibitor; py = person $\bullet y e a r s$

Table 2: Risk of Axis I diagnosis in HIV-infected persons, by demographics \& HIV factors, 2005 - Bethesda, Maryland.

\section{Discussion}

In this retrospective analysis of a cohort with unrestricted access to care, the incidence of an axis I mental health disorder was high at 3.1/100 py. However, since prescriptions for antidepressants/anxiolytics (25) or referrals to mental health (11) were not classified as MHD if a corresponding axis I diagnosis was not recorded, this incidence data most likely represents a minimum estimate.

With respect to demographic factors, risk of MHD found in the current study is most likely related to willingness of the individual to seek care and, therefore, likelihood of a diagnosis being made. Men and non-Caucasian persons are less likely to seek care for mood symptoms $[18,19]$. Further, officers may be more concerned about how a MHD might limit their career and choose not to seek care for their depression or anxiety symptoms. Diagnostic validity may also be a limiting factor, especially if examination or interview time to make the diagnosis is limited.

With respect to HIV specific factors, findings were somewhat unexpected: ART was a risk factor for any MHD and for depression, and the use of PI based ART was associated with higher risk of MHD compared to NNRTI based therapy; findings that may be explained by increasing use of PIs in the US. In 1996, the US ART prescription market share was $84 \%$ nucleoside reverse transcriptase inhibitor (NRTI), $16 \%$ PI, and $0.2 \%$ NNRTI. By 2000 , PI use increased to $57 \%$ NRTI, $28 \%$ PI, and $15 \%$ NNRTI; and by $2005,55 \%$ NRTI, $31 \%$ PI, and $13 \%$ NNRTI. Numbers of prescriptions for PIs steadily increased over time, generally exceeding those for NNRTIs by a factor of 2-3, during the period 1996-2005 [20]. Also since 1996, PIs are combined with NRTIs to treat HIV infection. AZT, the first antiretroviral drug used to treat HIV infection and the prototypical NRTI, is known to cause mitochondrial myopathy when used in high doses [21]. Similarly, other NRTIs have been shown to cause mitochondrial toxicity by inhibiting DNA polymerase $\gamma(\mathrm{Pol} \gamma)$ which is required for replication of mitochondrial DNA (mtDNA). Inhibition of Pol $\gamma$ causes depletion of mtDNA and apoptotic cell death [22]. Cellular efflux pumps, MRP4 and MRP5, are present on the plasma membrane, transport NRTIs out of the cell, and are inhibited by PIs, thus increasing intracellular NRTI concentration and toxicity [23]. Finally, mitochondrial dysfunction has been associated with bipolar disorder, schizophrenia, major depressive disorder, and other central nervous system diseases [24-27]. Therefore, increasing use of PIs may be responsible for enhanced NRTI toxicity and the trends noted in study data.

We acknowledge limitations inherent to a retrospective chart review, especially the non-randomized study design. Additionally, the heterogeneity of MHD made by different physicians and lack of use of strict DSM-IV criteria to arrive at a MHD are limitations. Although numbers of subjects developing MHD is small, especially those with anxiety, trends demonstrated across the three groups are consistent and, therefore, may represent a real effect of ART and deserve further exploration.

In conclusion, MHD remains a significant comorbidity among HIV infected persons and ART itself appears to increase risk of development of MHD. Historically, NNRTIs have been implicated in MHD; however, PIs may also negatively impact the mental health of HIV infected persons by increasing intracellular NRTI concentrations and subsequent mitochondrial damage. Clinicians must be aware of the importance of screening for and appropriate treatment of MHD in the management of HIV infection. Also, controlled, prospective studies examining ART use and MHD are needed and will improve 
Citation: Carpenter RJ, Riddle MS, White DA, Ganesan A (2012) Correlates of Depression among US Military Members Infected with the Human Immunodeficiency Virus. J AIDS Clinic Res 3:149. doi:10.4172/2155-6113.1000149

Page 4 of 4

management and quality of life of HIV infected persons by definitively defining ART risk related to MHD.

Conflict of Interest/Disclosure: There are no conflicts of interest to report. The authors have received no financial support for this manuscript.

Disclaimer: The views expressed in this manuscript are those of the authors and do not necessarily reflect the official policy or position of the Department of the Navy, Department of Defense, nor the U.S. Government.

\section{References}

1. Carr A, Samaras K, Thorisdottir A, Kaufmann GR, Chisholm DJ, et al. (1999) Diagnosis, prediction, and natural course of HIV-1 protease-inhibitor-associated lipodystrophy, hyperlipidaemia, and diabetes mellitus: a cohort study. Lancet 353: 2093-2099.

2. Fumaz CR, Tuldra A, Ferrer MJ, Paredes R, Bonjoch A, et al. (2002) Quality of life, emotional status, and adherence of HIV-1-infected patients treated with efavirenz versus protease inhibitor-containing regimens. J Acquir Immune Defic Syndr 29: 244-253.

3. Clifford DB, Evans S, Yang Y, Acosta EP, Goodkin K, et al. (2005) Impact of efavirenz on neuropsychological performance and symptoms in HIV-infected individuals. Ann Intern Med 143: 714-721.

4. Journot V, Chene G, De Castro N, Rancinan C, Cassuto JP, et al. (2006) Use of efavirenz is not associated with a higher risk of depressive disorders: a substudy of the randomized clinical trial ALIZE-ANRS 099. Clin Infect Dis 42: 1790-1799.

5. Perez-Molina JA (2002) Safety and tolerance of efavirenz in different antiretroviral regimens: results from a national multicenter prospective study in 1,033 HIV-infected patients. HIV Clin Trials 3: 279-286.

6. Brown GR, Rundell JR, McManis SE, Kendall SN, Zachary R, et al. (1992) Prevalence of psychiatric disorders in early stages of HIV infection. Psychosom Med 54: 588-601.

7. Ciesla JA, Roberts JE (2001) Meta-analysis of the relationship between HIV infection and risk for depressive disorders. Am J Psychiatry 158: 725-730.

8. Evans S, Ferrando S, Sewell M, Goggin K, Fishman B, et al. (1998) Pain and depression in HIV illness. Psychosomatics 39: 528-535.

9. Ferrando S, Evans S, Goggin K, Sewell M, Fishman B, et al. (1998) Fatigue in HIV illness: relationship to depression, physical limitations, and disability. Psychosom Med 60: 759-764.

10. Berg CJ, Michelson SE, Safren SA (2007) Behavioral aspects of HIV care: adherence, depression, substance use, and HIV-transmission behaviors. Infect Dis Clin North Am 21: 181-200, $x$.

11. Asch SM, Kilbourne AM, Gifford AL, Burnam MA, Turner B, et al. (2003) Underdiagnosis of depression in HIV: who are we missing? J Gen Intern Med 18: $450-460$.

12. Burack JH, Barrett DC, Stall RD, Chesney MA, Ekstrand ML, et al. (1993) Depressive symptoms and CD4 lymphocyte decline among HIV-infected men. JAMA 270: 2568-2573.

13. Leserman J, Petitto JM, Perkins DO, Folds JD, Golden RN, et al. (1997) Severe stress, depressive symptoms, and changes in lymphocyte subsets in human immunodeficiency virus-infected men. A 2-year follow-up study. Arch Gen Psychiatry 54: 279-285.

14. Starace F, Ammassari A, Trotta MP, Murri R, De Longis P, et al. (2002) Depression is a risk factor for suboptimal adherence to highly active antiretroviral therapy. $\mathrm{J}$ Acquir Immune Defic Syndr 31 Suppl 3: S136-139.

15. Judd FK, Cockram AM, Komiti A, Mijch AM, Hoy J, et al. (2000) Depressive symptoms reduced in individuals with HIVIAIDS treated with highly active antiretroviral therapy: a longitudinal study. Aust N Z J Psychiatry 34: 1015-1021.

16. Starace F, Bartoli L, Aloisi MS, Antinori A, Narciso P, et al. (2002) Cognitive and affective disorders associated to HIV infection in the HAART era: findings from the NeurolCONA study. Cognitive impairment and depression in HIVIAIDS. The NeurolCONA study. Acta Psychiatr Scand 106: 20-26.

17. Brodine SK, Starkey MJ, Shaffer RA, Ito SI, Tasker SA, et al. (2003) Diverse
HIV-1 subtypes and clinical, laboratory and behavioral factors in a recently infected US military cohort. AIDS 17: 2521-2527.

18. Alegria M, Chatterji P, Wells K, Cao Z, Chen CN, et al. (2008) Disparity in depression treatment among racial and ethnic minority populations in the United States. Psychiatr Serv 59: 1264-1272.

19. Branney P, White A (2008) Big boys don't cry: depression and men. Advances in Psychiatric Treatment 14: 256-262.

20. Jing Y, Klein P, Kelton CM, Li X, Guo JJ (2007) Utilization and spending trends for antiretroviral medications in the U.S. Medicaid program from 1991 to 2005. AIDS Res Ther 4: 22.

21. Dalakas MC, Illa I, Pezeshkpour GH, Laukaitis JP, Cohen B, et al. (1990) Mitochondrial myopathy caused by long-term zidovudine therapy. N Engl J Med 322: 1098-1105.

22. Kakuda TN (2000) Pharmacology of nucleoside and nucleotide reverse transcriptase inhibitor-induced mitochondrial toxicity. Clin Ther 22: 685-708.

23. Petit F, Fromenty B, Owen A, Estaquier J (2005) Mitochondria are sensors for HIV drugs. Trends Pharmacol Sci 26: 258-264

24. Finsterer $\mathrm{J}(2006)$ Central nervous system manifestations of mitochondria disorders. Acta Neurol Scand 114: 217-238.

25. Gardner A, Johansson A, Wibom R, Nennesmo I, von Dobeln U, et al. (2003) Alterations of mitochondrial function and correlations with personality traits in selected major depressive disorder patients. J Affect Disord 76: 55-68.

26. Munakata K, Iwamoto K, Bundo M, Kato T (2005) Mitochondrial DNA 3243A>G mutation and increased expression of LARS2 gene in the brains of patients with bipolar disorder and schizophrenia. Biol Psychiatry 57: 525-532.

27. Rezin GT, Amboni G, Zugno Al, Quevedo J, Streck EL (2009) Mitochondria dysfunction and psychiatric disorders. Neurochem Res 34: 1021-1029. 